\title{
Computational models for the in silico analysis of drug delivery from drug-eluting stents
}

\author{
"In silico tests facilitate the study of drug distribution and deposition, providing \\ results that could not otherwise be gathered..."
}

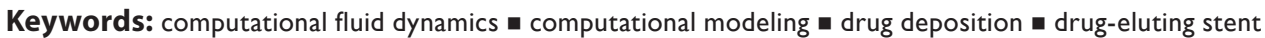
- local drug delivery $\mathbf{n}$ restenosis post-stenting
\end{abstract}

Stents are tubular meshed devices implanted to restore the perviety of an occluded vessel owing to the presence of an atherosclerotic plaque. These devices were introduced in to clinical practice from 1980. The first stent implanted in a human coronary artery was the Wallstent [1], a self-expandable metallic device. The use of a stent to expand the vessel was introduced to overcome the greater limit of angioplasty, the elastic recoil of the vessel wall, yet also caused the onset of another, different pathology: intra-stent restenosis. This pathology results from injuries on the vessel wall after balloon inflation as well as the different fluid dynamic regime established after stent implantation [2]. Intra-stent restenosis is caused by the abnormal growth of tissues within stent meshes, leading to the implant failure.

The common therapeutic approach to limit hyperplasia is the systemic administration of antimitotic and anti-inflammatory drugs. This treatment generally fails because effective dosing levels have toxic effects on patients. Since 2000, a new and emerging class of stents was introduced to redress this problem. We are referring to drug-eluting stents (DES), new devices loaded with one or more active principles for the local administration of the drug, avoiding the systemic administration of massive doses. DES are metallic devices impregnated with a drug on their surface or coated with a polymeric thin layer containing the active principle.

\section{Concerns related to DES implantation}

The introduction of the polymeric coating caused several problems related to hemo/cytocompatability. However, the major challenge is to obtain appropriate drug delivery kinetics to justify the introduction of the polymeric component in an already complex and difficult to manage system. For these devices it is fundamental to evaluate three important aspects with respect to drug delivery: if the amount of drug eluted is comprised within the therapeutic range of administration; if the drug distribution reaches a sufficient area; and if the drug-elution time is sufficient to achieve the required biological effects. The increase in DES complexity on introduction of a polymeric coating is justified if the coating can address the three aforementioned aspects of drug-delivery kinetics.

The evaluation of DES effectiveness during the preclinical development is tricky question because it is not possible to quantify in vivo the amount of drug actually released within tissues without animal testing and sacrifice of the animal. In addition, in vitro tests to evaluate drug-delivery kinetics are affected by several limitations, such as the difficulty of recreating the biological environment. In vitro and ex vivo tests are able to furnish results concerning diffusion of a drug across the polymeric coating and the arterial wall, but all phenomena related to the solvent-driven flow across the wall cannot be analyzed.

A promising method to evaluate drug-delivery properties of DESs is represented by in silico analysis, based on the use of mathematical models to predict the behavior and efficacy of these devices. Computational modeling offers the possibility to evaluate a series of data that cannot otherwise be acquired, providing a better understanding of the drug-delivery process. In silico tests allow for the evaluation of drug diffusion, transmural convective flow, dissolution of the active principle in the blood flow and metabolism, providing the user with easy access to interpretable drugdistribution maps versus time.

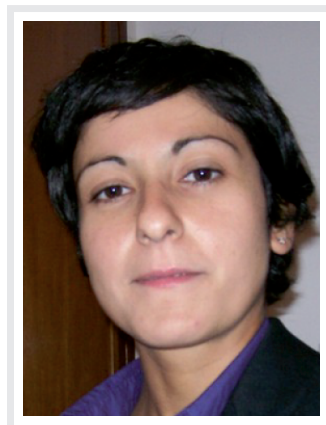

Mariacristina Gagliardi

Deptment of Chemical Engineering, Industrial Chemistry and Materials Science, University of Pisa, Largo Lucio Lazzarino I, 56126, Pisa, Italy E-mail: m.gagliardi@diccism.unipi.it 


\section{Relevant in silico studies of drug delivery from DESs}

Several computational models were studied to analyze drug delivery from DESs, developing ad hoc transient mass-transfer models, both in 2D [3-5] and 3D [6-8].

Drug-delivery phenomena are fluid dynamic and species transport problems; the science related to these topics is computational fluid dynamics (CFD). A simple 2D CFD analysis of drug transport toward biological tissues is reported by Hwang et al. [3]. In this work, drug distribution was easily studied using a simplified method based on the transient diffusion model for the transport toward the arterial wall, in addition to a transient diffusion-convection model to study drug dissolution in the blood. In this case only Fick's laws were used to describe the behavior of the arterial wall, taking into account the dependencies of diffusion on direction. This study examined the limitations of the efficacy of drug delivery from a DES relating to local pharmacokinetics, underlining a strong dependency on the balance between convective and diffusive flow. Following this, a similar model was proposed [4], which introduced thrombus formation. This work reported the analysis of the effect of thrombus size on drug deposition. The 2D computational model developed was able to predict fluctuations in drug deposition revealing that diffused thrombi increased the arterial drug deposition, reducing drug washout and consequently increasing the drug availability. This was a relevant discovery because drugs generally eluted from stents are toxic if administered over a specific dose. Evaluation of the causes of fluctuations in drug deposition can improve understanding of the effective local drug elution. A more recent study also considered the presence of a polymeric coating loaded with a drug and the effect of local hemodynamics on drug delivery, by analyzing drug release with a multidomain approach [5]. This work focused attention on the differences in drug distribution due to the stent structure, strongly influencing convective drug flow.

However, the analysis of drug distribution can be more interesting if studied through a 3D model. Three dimensional computational analyses of drug delivery and distribution from DESs are performed using computational models describing the geometries of device and biological tissues and all characteristics related to diffusion, advection and dissolution of the drug. In recent years, several studies have been carried out to analyze drug elution from DESs using different 3D models. The use of computational models to study drug distribution can be very useful for analyzing geometrically complex problems, such as the drug distribution from DESs implanted in bifurcated vessels, where complex architecture and the mechanical and fluid-dynamic environment compound the lesion heterogeneity. In the work by Kolachalama et al. [6] this analysis was carried out comparing results obtained from bifurcated and nonbifurcated vessels. The more relevant discovery was that an asymmetric drug deposition occurred due to the local flow related not only to the anisotropic stent design but also to asymmetries in the vessel. Also in this case the visualization of drug distribution can help predict the possibility of the occurrence of dosages over the toxicity level.

A detailed drug-delivery analysis using CFD on a polymer-coated Cordis BX-Velocity Stent (Johnson \& Johnson) was carried out by Zunino et al. [7], demonstrating that drug release into the lumen was not significant. This analysis only reported results related to the first hour after the implant. Thereafter the model is not suitable to verify whether the drug concentration is within the therapeutic range for the necessary time of pharmacological administration. The possibility of visualizing the arterial drug distribution pattern for a long period after stent implantation allows the evaluation of the effective therapeutic action of the device. In addition, the possibility to compare different stent models, coatings and starting drug loads can be an interesting approach for a better understanding of the pharmacological action of DESs. This comparative analysis was recently carried out by approaching the problem on the basis of the analogy between thermal and mass transfer [8]. Three different commercial devices were analyzed by testing the drug-elution behavior from acrylic coatings loaded with three different starting percentages of paclitaxel, an antimitotic drug used to load several commercial DESs. In Figure I some interesting results of this study are reported. Unlike previously cited works, the analysis on specific polymeric coatings was carried out using results obtained by in vitro tests as input data. In this case, the analysis represents an a posteriori study for the verification of DES performance. However, computational models can be used to design the stent geometry, the properties of polymeric coatings (in terms of chemical composition, porosity and thickness, major parameters affecting drug-delivery kinetics) and starting drug load with an a priori approach. 


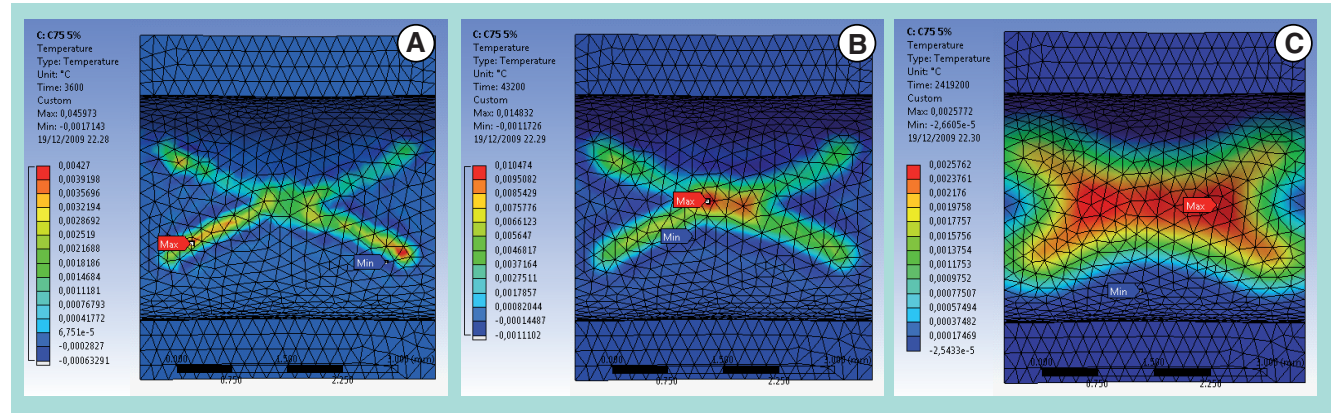

Figure 1. Concentration of paclitaxel (w/w) within arterial wall, (A) after $1 \mathrm{~h},(B) 1$ week and (C) 1 month, eluted from Cypher ${ }^{\circledR}$ Stent (Johnson \& Johnson, NJ, USA) coated with copolymer poly (methylmethacrylate-co-butylmethacrylate) in percentage molar ratio 75:25 (starting drug load was $5 \% \mathrm{w} / \mathrm{w}$ ).

Results were obtained using ANSYS ${ }^{\circledR}$ Workbench 12.0 (Ansys Inc., PA, USA).

\section{Conclusion}

Design, development, testing, optimization and verification of DES performance can be well supported by computer-assisted methods to limit extensive trial and error experiments as well as for a deeper understanding of the therapeutic action of DES. In silico tests facilitate the study of drug distribution and deposition, providing results that could not otherwise be gathered through different tests, also representing a valid approach for the selection of appropriate design parameters and leading to a strong reduction of development time and costs. Computational models can be used to predict whether drug concentration within tissues is within the therapeutic range, if the area where lesion occurs is completely affected by drug administration and if the drug can be eluted with sustained kinetics, to obtain optimal localized pharmacological therapy. Safer stents can be designed using results obtained through computational modeling, even if in vitro, ex vivo and in vivo tests remain strongly necessary to validate in silico models.

\section{Financial \& competing interests disclosure}

The author has no relevant affiliations or financial involvement with any organization or entity with a financial interest in or financial conflict with the subject matter or materials discussed in the manuscript. This includes employment, consultancies, honoraria, stock ownership or options, expert testimony, grants or patents received or pending, or royalties.

No writing assistance was utilized in the production of this manuscript.

\section{Bibliography}

1 Haase KK, Karsch KR. Coronary stents implantation of foreign bodies into stenotic human coronary arteries: dream or nightmare? Eur. Heart J. 18(4), 552-553 (1997).

2 Frank OA, Walsh PW, Moore JE Jr. Computational fluid dynamics and stent design. Artif. Organs 26(7), 614-621 (2002).

3 Hwang WC, Wu D, Edelman ER. Physiological transport forces govern drug distribution for stent-based delivery. Circulation 104, 600-605 (2001).
4 Balakrishnan B, Dooley FJ, Kopia G, Edelman ER. Thrombus causes fluctuations in arterial drug delivery from intravascular stents. J. Control. Release 131, 173-180 (2008).

5 Vairo G, Cioffi M, Cottone R, Dubini G, Migliavacca F. Drug release from coronary eluting stents: a multidomain approach. J. Biomech. 43(8), 1580-1589 (2010).

6 Kolachalama BV, Levine GE, Edelman ER. Luminal flow amplifies stent-based drug deposition in arterial bifurcations. PLOS ONE 4(12), E8105 (2009).
7 Zunino P, D’Angelo C, Petrini L, Vergara C, Capelli C, Migliavacca F. Numerical simulation of drug eluting coronary stents: mechanics, fluid dynamics and drug release. Comput. Methods Appl. Mech. Eng. 198(45-46), 3633-3644 (2009).

8 Gagliardi M, Silvestri D, Cristallini C. Modeling drug delivery from stents through a finite element analysis. Presented at: 23rd European Conference on Biomaterials. Tampere, FL, USA, 11-15 September 2010 (Abstract 3664). 